IEEE Instrumentation and Measurement

Technology Conference

Brussels, Belgium, June 4-6, 1996

\title{
A Fourth-Order BandPass $\Sigma \Delta$ Modulator Using Current-Mode Analog/Digital Circuits
}

\author{
J.M. de la Rosa, B. Pérez-Verdú, F. Medeiro, and A. Rodríguez-Vázquez \\ Dept. of Analog Design, Centro Nacional de Microelectrónica (CNM), \\ Ed. CICA, Av. Reina Mercedes s/n, 41012 Sevilla, SPAIN. \\ Phone: (34)-5-4239923, Fax: (34)-5-4231832, E-mail: angel@cnm.us.es
}

\begin{abstract}
We present a fourth-order bandpass $\Sigma \Delta$ switchedcurrent modulator $1 C$ in $0.8 \mu \mathrm{m}$ CMOS single-poly technology. Its architecture is obtained by applying a lowpass to bandpass transformation $\left(z^{-1} \rightarrow-z^{-2}\right)$ to a second-order lowpass modulator. It has been realized using fully-differential circuitry with common-mode feedback. Measurements show $B$ bit dynamic range up to $5 \mathrm{MHz}$ clock frequency and $10 \mathrm{KHz}$ bandwidth.
\end{abstract}

\section{INTRODUCTION}

$\Sigma \Delta$ converters use oversampling to reduce the in-band power of quantization noise, and feedback to shape this noise and push it out of band. The intrinsic tolerance of $\Sigma \Delta$ converters to analog circuitry inaccuracies renders them very well-suited for the on-chip design of high-resolution interfaces in mixed-signal ASICs.

Applications of $\Sigma \Delta$ converter ICs span from instrumentation to telecommunications. In particular, bandpass $\Sigma \Delta$ s are used at the front-end of modern narrowband communication systems [1][2]. Table 1 summarizes the state-of-the-art on $\Sigma \Delta$ BandPass realizations. [3] and [4] are in BiCMOS technology. The former use "off-chip" LC resonators, while the latter employs sampled-data resonators realized with switched capacitor circuits. Similar design technique is employed in [5] and [6], in CMOS technology. The table displays the center frequencies and bandwidths measured for each realization. They are suitable for broadcast and voice AM, IS-54 cellular telephony, and GSM telephony. However, they are not the best suited for standard VLSI where neither accurate thermal oxide linear capacitors nor high $f_{T}$ BJT transistors are available.

The trend towards combination of analog and digital circuitry on mixed-signal chips with low production cost has motivated exploring design techniques such as SwitchedCurrents (SI), which can be manufactured in standard VLSI single-poly technologies without any extra processing steps [7].
SI circuits have already been used for lowpass $\Sigma \Delta s[8,9]$. To the best of our knowledge, this paper reports the first IC realization of an SI bandpass $\Sigma \Delta \mathrm{IC}$. It is a fourth-order architecture and has been designed using fully-differential circuitry in $0.8 \mu \mathrm{m}$ CMOS single-poly technology. Measurements show 8 bit dynamic range up to $5 \mathrm{MHz}$ clock frequency. Since the commercial AM broadcast band is 540 to $1600 \mathrm{KHz}$, with stations occupying a band $10 \mathrm{KHz}$ wide, this modulator performs according to digital AM receiver requirements [6].

\section{ARCHITECTURE OF THE MODULATOR}

The modulator architecture has been obtained by applying a $z^{-1} \rightarrow-z^{-2}$ transformation to the second-order lowpass prototype of Fig. 1 [5]. This transformation maintains the stability properties of the lowpass prototype, whose practical use has been demonstrated in both SC and $\mathrm{SI}$ implementations [8].

Because of this transformation, the integrators in the lowpass prototype become resonators with transfer function,

Table 1: State of the Art of BandPass $\Sigma \Delta$ Modulators

\begin{tabular}{|c|c|c|c|c|}
\hline Reference & $\begin{array}{c}F_{\mathrm{s}} \\
(\mathrm{MHz})\end{array}$ & $\begin{array}{c}\mathrm{BW} \\
(\mathrm{KHz})\end{array}$ & $\begin{array}{l}\text { SNR } \\
\text { (dB) }\end{array}$ & Technology \\
\hline $\begin{array}{l}\text { Jantzi[6] } \\
\text { (1993) }\end{array}$ & 1.852 & 8 & 63 & $\begin{array}{l}\text { CMOS } 3 \mu \mathrm{m} \\
\text { double poly }\end{array}$ \\
\hline $\begin{array}{l}\text { Longo[5] } \\
\text { (1993) }\end{array}$ & 7.2 & 30 & 92 & $\begin{array}{l}\text { CMOS } 1 \mu \mathrm{m} \\
\text { double poly }\end{array}$ \\
\hline $\begin{array}{l}\text { Troster[3] } \\
\text { (1993) }\end{array}$ & 26 & 200 & 55 & $\begin{array}{l}\mathrm{BiCMOS} \\
1.2 \mu \mathrm{m} / 7 \mathrm{GHz} \\
\text { off-chip LC }\end{array}$ \\
\hline $\begin{array}{l}\text { Singor[4] } \\
\text { (1995) }\end{array}$ & 42.8 & 200 & 57 & $\begin{array}{l}\text { BiCMOS } \\
0.8 \mu \mathrm{m}\end{array}$ \\
\hline
\end{tabular}




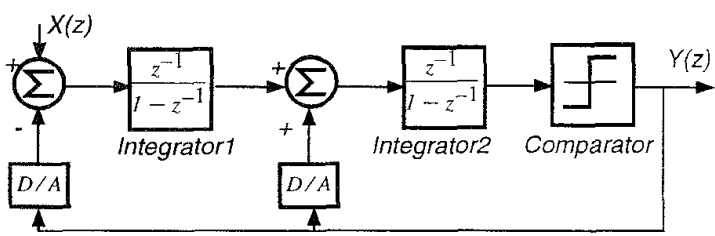

Fig. 1: Initial lowpass block diagram

$$
R(z)=\frac{-z^{-2}}{1+z^{-2}}
$$

Fig. 2 shows two alternative structures to realize $R(z)$ above in a way compatible with SI design technique. Structure -1 is a cascade of one Forward-Euler integrator and one Forward-Euler differenciator. Structure-2 is a cascade of two Back-Euler integrator blocks.

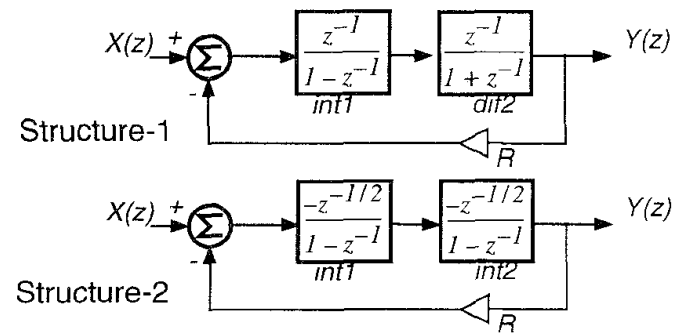

Fig. 2: Alternative structures to implement the resonator block

For both structures in Fig. 2, the riominal value of the feedback coefficient $R$ is 2 . In practical realizations, changes in this coefficient degrade the quality of the resonator. Fig. 3 displays the pole movement with $R$ varying around its nominal value, for both structures. It is seen that the poles remain in the circle unit for Structure-2 -- the reason leading us to choose this structure to design the modulator.

Fig. 4 shows the block diagram of the bandpass modulator. The required feedback delay has been realized through two additional delay blocks, as depicted in the figure. Assuming that the 1 bit quantizer in the resulting bandpass modulator can be modelled as an additional white noise source with effective gain of $k$, the output is given as,

$$
Y(z)=z^{-2} X(z)+\left(1+z^{-2}\right)^{2} E(z)
$$

where $X(z)$ is the modulator input signal, $Y(z)$ is the modulator output, and $E(z)$ is the additional quantization noise source in the linear model. We see that the transfer

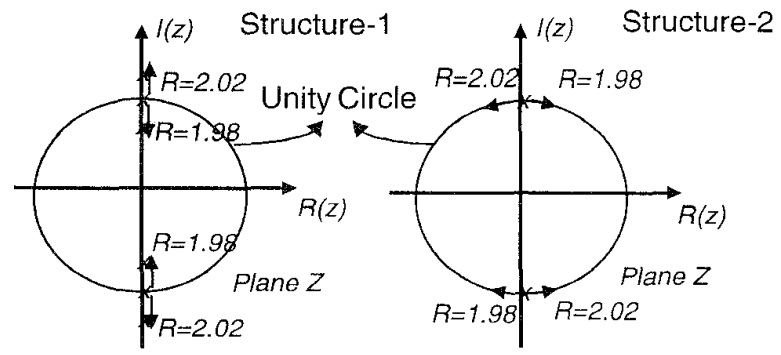

Fig. 3: Displacement of poles with $R$.

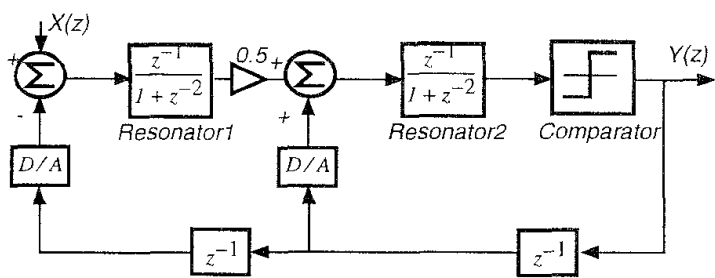

Fig. 4: Block Diagram of the Modulator.

function for the input is a double-delay; on the other hand, the transfer function $N T F(z)$ for the quantization noise has two transmission zeroes at $f_{s} / 4$. This is the equivalent of the two zeroes at DC observed in the lowpass prototype.

Fig. 5 shows simulation results of the modulator architecture obtained using ASIDES [10], a proprietary behavioral simulator for $\Sigma \Delta$ modulators. Fig. 5 a shows an FFT of the simulated modulator output for a sinusoidal input of $-6 \mathrm{~dB}$ range, centered on $2.5 \mathrm{MHz}$, and a clock frequency of $10 \mathrm{MHz}$. The use of a scaling factor 0.5 at the output of the first modulator obtains similar dynamic range at the output of both resonators, as shown in the histogram of Fig. 5b. Thus both resonators can be designed identically.

\section{DESIGN PROCESS}

The methodology used to design the modulator is based on our previous work in [10]. The coefficients of the modulator are chosen through a statistical optimization procedure where the cost function is built using equations to describe the major non-idealities of SI blocks (namely, memory cells and integrators) that degrade the performance bandpass $\Sigma \Delta$ modulators. As regards to the calculation of the dimensions of the transistors in the SI blocks we have used also statistical optimization, and electrical simulations to evaluate the cost function.

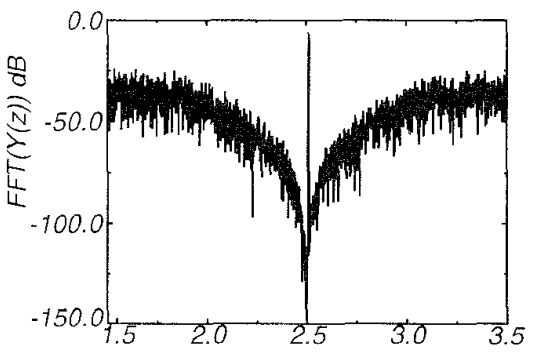

a) Frequency $(\mathrm{MHz})$

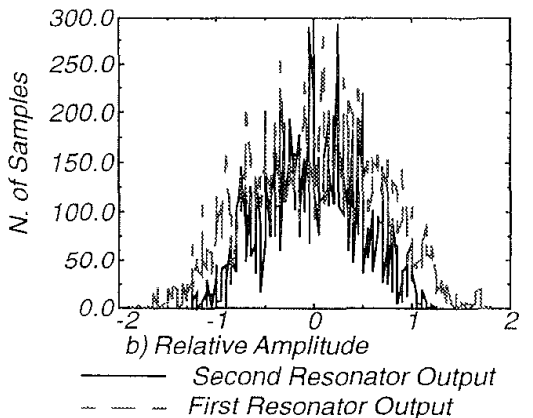

Fig. 5: Behavior Simulations of the Modulator 


\section{A.Quantization noise}

Previous analysis of SI memory cells and integrators have considered finite output-input conductance ratio $\left(\varepsilon_{g}\right)$, charge injection $\left(\varepsilon_{q}\right)$ and settling errors $\left(\varepsilon_{s}\right)$ [7]. For our purposes, we have also taken into account errors due to changes in the feedback loop gain,

$$
\varepsilon_{R}=1-\frac{R}{R_{N O M}}
$$

where $R_{N O M}$ is the nominal value of 2 . The transfer function of quantization noise when these non-idealities are taken into account is given by,

$$
N T F(z) \approx\left(1+z^{-2}\right)^{-2}+\varepsilon_{g}(z)+\varepsilon_{q}(z)+\varepsilon_{s}(z)+\varepsilon_{R}(z)
$$

where,

$$
\begin{aligned}
& \varepsilon_{g}(z)=\varepsilon_{q}(z)=\left(-4 \varepsilon_{g}\left(1+z^{-2}\right)\left(1-z^{-1}\right)\right) \\
& \varepsilon_{s}(z)=-4 \varepsilon_{s}\left(1+z^{-2}\right)\left(20-16 z^{-1}\right) \\
& \varepsilon_{R}(z)=-12 \varepsilon_{R^{z}}^{2}\left(1+\frac{4}{3} z^{-2}\right)
\end{aligned}
$$

It is seen that the zeroes of NTF(z) are shifted from their nominal positions at $f_{s} / 4$, thereby degrading the filtering performed by the resonators. Each non-ideality produces different amount of degradation. As designers, we would like to have insight about the separate influence of each nonideality. Fig. 6 illustrates this. There we plot three different families of Bode diagrams for $|N T F(f)|$ with $z=\exp (\mathrm{j} 2 \pi f / f s)$. Each has been obtained by keeping only one error term and nulling the others. It is seen that, for similar error magnitude, the most important shifting in $|N T F(f)|$ is due to settlling error $\varepsilon_{s}$. On the other hand, the gain error $\varepsilon_{R}$ manifests only through shiftings of the signal band around the center frequency.

The quantization noise power $P_{E Q}$ can be obtained by integrating the power spectral density in the signal band,

$$
P_{E Q}=\frac{\Delta^{2}}{12 f_{s}} \int_{\left(f_{s} / 4-(B W) / 2\right)}^{\left(f_{s} / 4+(B W) / 2\right)}|N T F(f)|^{2} d f
$$

Where $\Delta$ is the quantization step and $B W$ is the information signal band. After some algebra, and assuming that errors are $<1 \%$ (for simplifications) one obtains,

$$
P_{E Q}=\frac{\Delta^{2}}{120} \frac{\pi^{4}}{M^{5}}+\frac{\Delta^{2}}{9} \frac{\pi^{2}}{M^{3}}\left(4 \varepsilon_{g}^{2}+4 \varepsilon_{q}^{2}+16 \varepsilon_{s}^{2}+3 \varepsilon_{R}^{2}\right)
$$

where $M$ is the oversampling ratio defined as $f_{s} /(2 B W)$. The first term in (6) is the ideal $P_{E Q}$ and the others are the error contributions
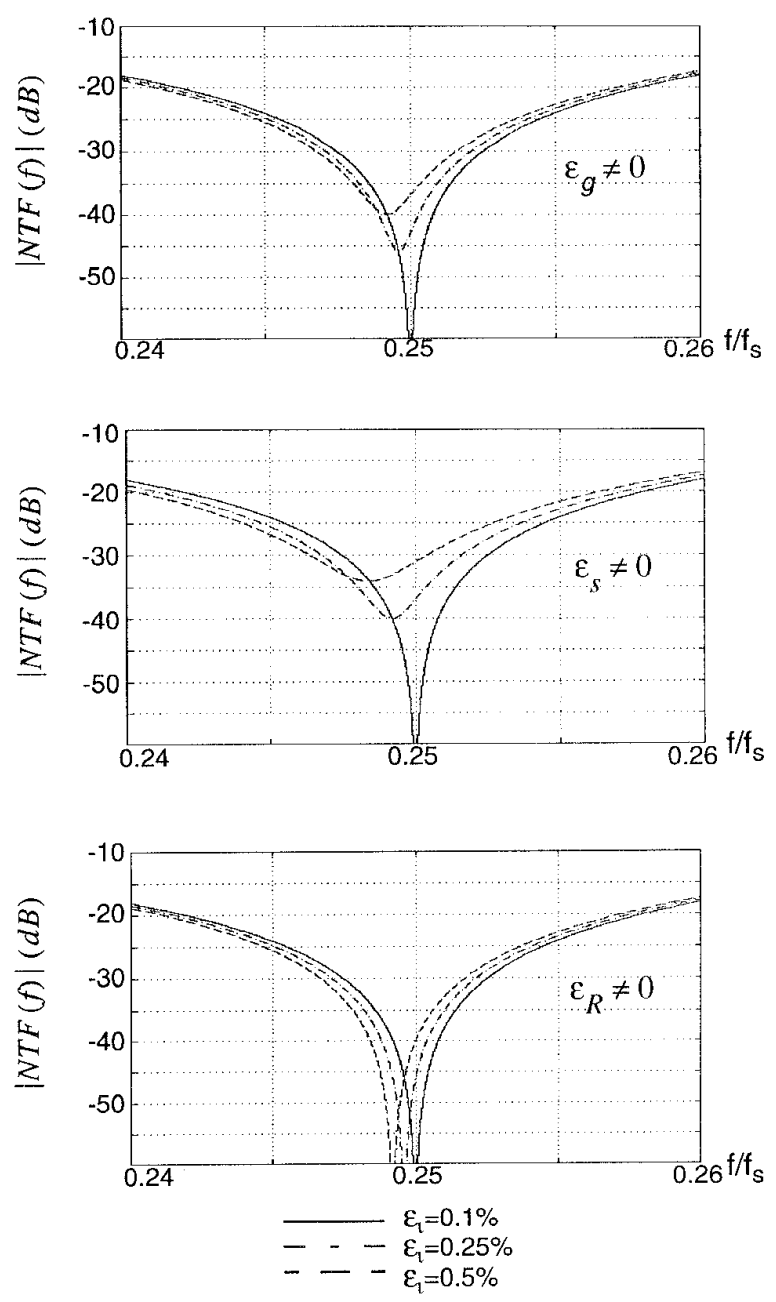

Fig. 6: Influence of non-idealities on NTF(z)

\section{B.Thermal Noise}

Thermal noise is the ultimate limiting factor in practical realizations of $\Sigma \Delta$ modulators. The most important contribution is due to the first resonator in Fig. 4, whose inputreferred current noise Power Spectral Density $(P S D)$ is given by [11],

$$
S_{I}(f)=\left(\frac{\tau}{T}\right)^{2} \operatorname{Sinc} c^{2}(f \tau) S_{o} \frac{2 B W_{n}}{f_{c k}}
$$

where $B W_{n}$ is the noise equivalent bandwidth and $S_{0}$ is the thermal noise $P S D$ before sampling.

The maximum signal to noise ratio for a sinusoidal input of $\Delta$ amplitude is given as,

$$
S N R=\frac{\Delta^{2} / 2}{P_{E Q}+P_{T H}}
$$

where $P_{T H}$ is the thermal noise power obtained after integration of ( 7$)$ in the signal band. 


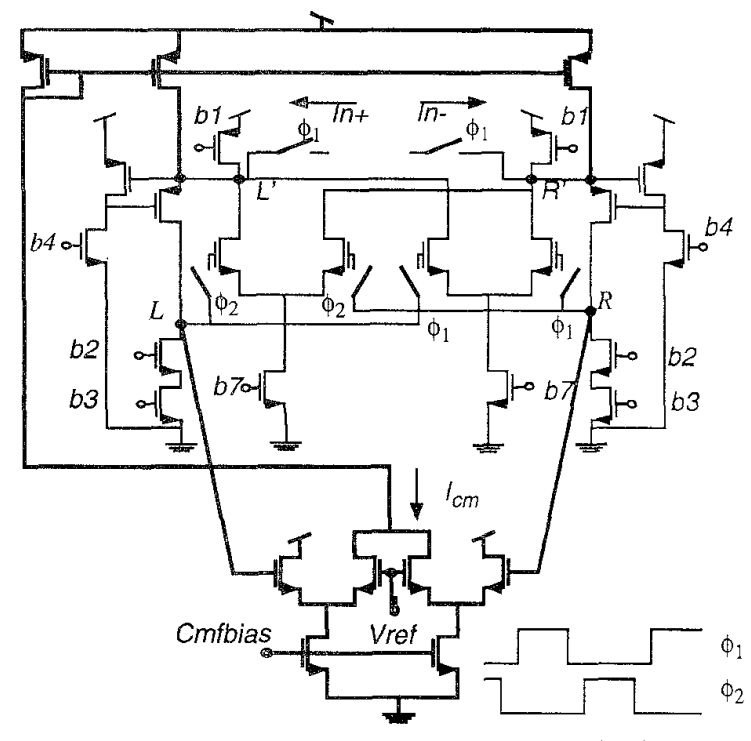

Fig. 7: Regulated Folded-Cascode Integrator with CMFB

\section{MODULATOR BLOCKS}

\section{A.SI Integrator}

Fig. 7 shows the implementation of the integrator with SI techniques. For maximum reduction of errors, we used a fully-differential regulated folded cascode structure .. inspired in [11]. The thin line in this figure represents the signal path, while the thick lines represent the CommonMode Feedback path (CMFB). The cell incorporates local feedback in the signal path to increase the input conductance and thus reduce the interconnection loading errors. Minimum size dummy transistors are used to attenuate feedthrough cancellation. Besides, the differential structure of the cell provides first-order cancellation of the residual feedthrough. To avoid transient spikes, the cell output nodes are connected to low-impedance nodes while the steering switches are open. Also, delayed versions have been used for the clock phases that control the steering switches.

\section{B.1-bit D/A Converter}

Fig. 8 shows a schematic of the 1-bit D/A converter used in the circuit, consisting of a current source controlled by the comparator output [8]. The stacked cascode current mirror

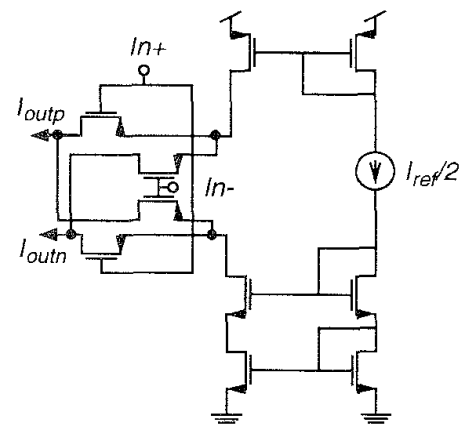

Fig. 8: 1-bit $\mathrm{D} / \mathrm{A}$ Converter.

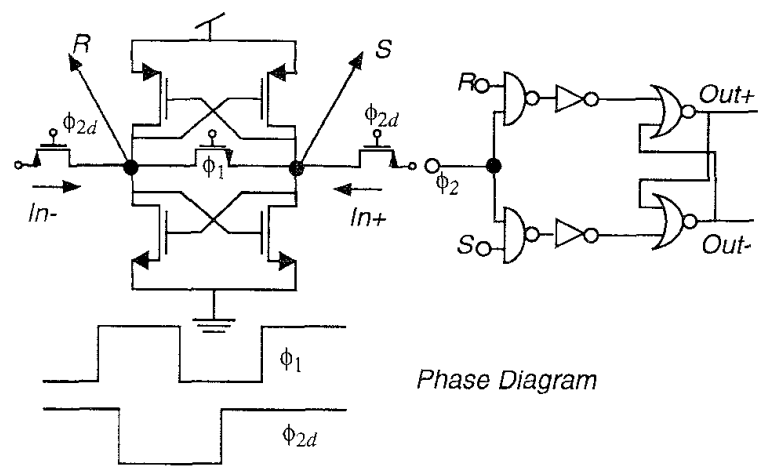

Fig. 9: Block diagram of the Current Quantizer

has been chosen because it resembles the structure of the cell memory. The two output currents change the flow direction depending on the comparator output. A small offset is no serious problem for the modulator. PMOS switches are used due to the input voltage range required in the integrators.

\section{C.1-bit Quantizer}

Fig. 9 shows the quantizer block diagram, made up of a regenerative latch [13] and an $\mathrm{RS}$ flip-flop that maintains the output value in the phase $\phi_{1}$, which is the phase where the resonators are feedback.

The regenerative latch operates as follows. Since the input switches are PMOS, currents are sampled in the complementary phase $\phi_{2 d}$, developing a small difference voltage through the NMOS switch. When the NMOS switch opens, the current which flows through it cuts off abruptly, and the latch impedance changes from positive to negative (due to the positive feedback). Consequently, depending on the sign of the small difference of stored voltage, the latch output nodes ( $R$ and $S$ ) will go to the positive or negative rail. A fast comparison is thus obtained with low input current levels.

\section{D.Input Buffer}

If the voltage-to-current conversion needed at the modulator output is realized using an external resistance, the transient behavior of resonator- 1 is influenced by the pad capacitance. To avoid this, an input buffer is included in our prototype. Fig. 10 shows the schematics of this block. Input to this block can be either fully differential or single-ended,

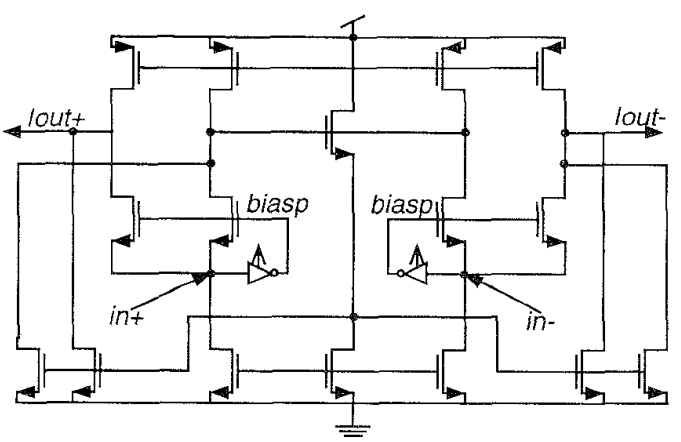

Fig. 10: Input Buffer 

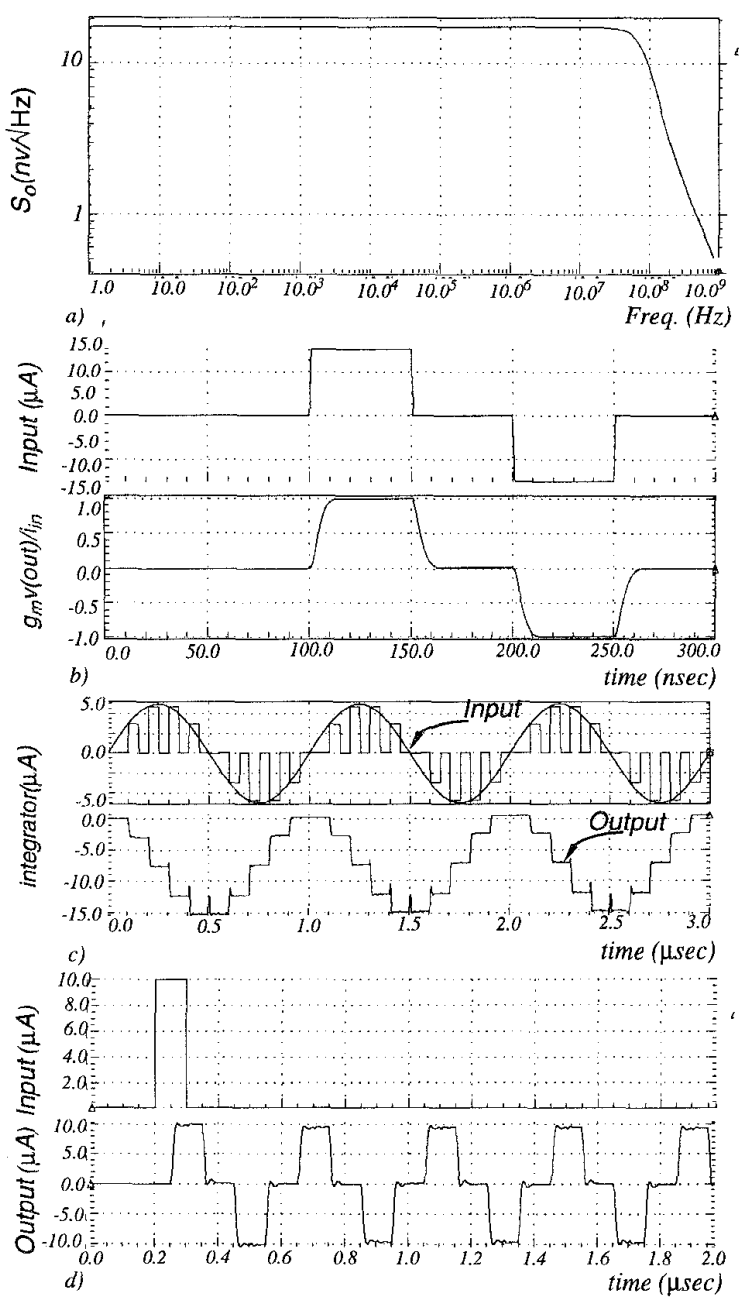

Fig. 11: HSPICE Simulations of the Memory Cell and Resonator.

while output is always fully-differential. The common-mode gain of the blocks is significantly smaller than the differential gain. Any remaining common mode will be rejected by the CMFB circuit of the differential SI integrator.

\section{SIMULATION RESULTS}

Fig. 11 shows several simulations of the modulator blocks using HSPICE. Fig. 11a shows the total noise rms voltage $\left(S_{o}\right)$ of the buffer and integrator cascade. This AC simulation is made with all switches closed and $1 / f-$-noise removed and results in an equivalent noise PSD of $17.9 \mathrm{nV} / \mathrm{JHz}$ at the gates of the memory transistors of the integrator. Fig. 11b shows transient simulation of this cell in the storage phase; $v($ out $)$ is the gate voltage of the memory transistor. Fig. 110 shows the time response of the integrator to a sinusoidal input of $5 \mu \mathrm{A}$ range and $1 \mathrm{MHz}$ when sampled to $10 \mathrm{MHz}$. Finally, Fig. 11d shows a simulation of the time response of the resonator when it is excited by an impulse signal of $10 \mu \mathrm{A}$ range. The Resonator output should repeat the values in the ranges $10 \mu \mathrm{A}, 0,-10 \mu \mathrm{A}, 0$ periodically. The observed

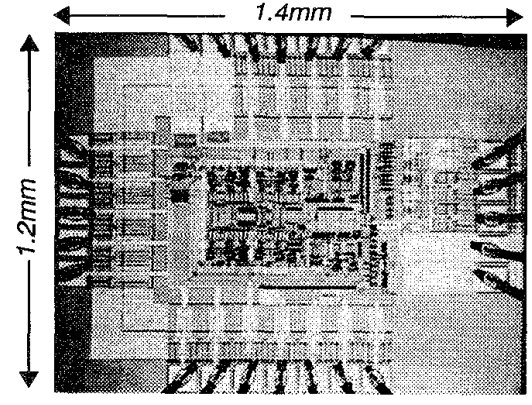

Fig. 12: Chip microphotograph

attenuation is due to non-idealities.

\section{EXPERIMENTAL RESULTS}

Fig. 12 is a microphotograph of the prototype, which has been designed in a CMOS $0.8 \mu \mathrm{m}$ single-poly technology. It occupies $0.79 \times 0.54 \mathrm{~mm}^{2}$ (without the interconnection pads), and consumes $15 \mathrm{~mW}$ from a $5 \mathrm{~V}$ supply. A clock phase generator is also included.

\section{A.Input Buffer}

Table 2 summarizes measurements made on an isolated buffer using a single-ended input. The white output noise has been measured with two external resistances connected to a $4 \mathrm{~V}$ reference voltage. $\mathrm{DC}$ measurements have been realized with the semiconductor parameter analyzer HP4145, while bandwidth have been measured with the network analyzer HP4195.

In order not to constraint the high-frequency performance of the buffer, an external loading device with large bandwidth has been used. Fig. 13 shows the measurement set-up. It is based on the AD844, a high speed monolithic operational amplifier implemented as a second generation current conveyor of gain +1 (CCll + ) [14]. Fig. 14 shows an experimental Bode plot corresponding to $C_{P A D}=11 \mathrm{pF}$ (the whole parasitic capacitance in the experimental board). The measured $3 \mathrm{~dB}$ frequency is $28 \mathrm{MHz}$, which results approximately equal to the dominant pole $\left(2 \pi R_{i n} C_{P A D}\right)^{-1}$, created by the input resistance of the buffer.

\section{B.Modulator}

A test board was fabricated following the indications in [15]. Fig. 15(a) shows the measured power spectrum of the modulator output when the clock frequency is $5 \mathrm{MHz}$ and the input is a $-6 \mathrm{~dB} @ 1.26 \mathrm{MHz}$ single tone. In order to obtain the signal to noise ratio, bit streams were obtained for the modulator at different signal levels by using the HP82000

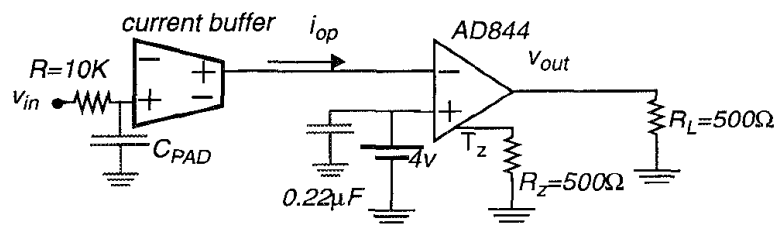

Fig. 13: AC Buffer Measurement Set-Up 


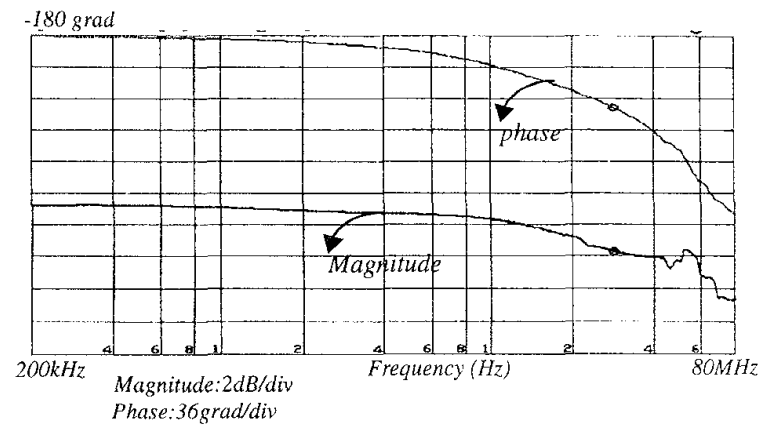

Fig. 14: Experimental Bode plot

data acquisition equipment. Hanning-windowed 32768-point FFTs were performed on each of those bit streams and SNR was calculated from them by varying signal levels. Fig. 15(b) shows the measured SNR versus input for the same modulator evaluated in a bandwidth of $\pm 5 \mathrm{KHz}$ around the input frequency.

\section{CONCLUSIONS}

A SI bandpass $\Sigma \Delta$ modulator has been designed in a CMOS $0.8 \mu \mathrm{m}$ single-poly technology. The architecture is obtained by applying a lowpass to bandpass transformation to a second order lowpass architecture. The circuit has been realized using switched-c̈urrent fully differential regulated cascode cells. Measurements show 8 bit dynamic range up to $5 \mathrm{MHz}$ clock frequency. Since the commercial AM broadcast band is 540 to $1600 \mathrm{Khz}$, with stations occupying a band $10 \mathrm{KHz}$ wide, this modulator performs according to digital AM receiver requirements [6].

\section{REFERENCES}

[1] A.M. Thurston, T.H. Pearce, M.D. Higman and M.J. Hawkstord "Bandpass Sigma Delta A-D Conversion," Workshop on Advances in Analog Circuit Design, pp. 267-297, April 1992.

[2] S Jantzi, Schreier and M. Snelgrove, "Bandpass Sigma-Delta Analog-to-Digital Conversion," IEEE Trans. Circuits and Systems, Vol. 31, pp. 1406-1409, November 1991.

[3] G. Troster, P. Sieber, K. Schoppe, A. Wedel, E. Zocher and J. Arndt, "An interpolative bandpass converter on a $1.2 \mu \mathrm{m} B i C M O S$ analog/digital array," IEEE J. Solid State Circuits, Vol. 28, pp.

Table 2: Measurements of the current buffer

\begin{tabular}{|c|c|c|c|}
\hline PARAM. & HSPICE & Experimental & Unit. \\
\hline Diff. DC-Gain & 0.48 & 0.49 & -- \\
\hline Output swing & \pm 20 & \pm 12 & $\mu \mathrm{A}$ \\
\hline$R_{\text {in }}$ & 684 & $<684$ & $\Omega$ \\
\hline$R_{\text {out }}$ & 176 & 113 & $\mathrm{~K} \Omega$ \\
\hline $\begin{array}{c}\text { Common DC- } \\
\text { Gain }\end{array}$ & 0.03 & 0.03 & $\mathrm{~dB}$ \\
\hline $\begin{array}{c}\text { White output } \\
\text { Noise }\end{array}$ & 10 & 50 & $\mathrm{nV} / \mathrm{NHz}$ \\
\hline Bandwidth & 25 & $>20$ & $\mathrm{MHz}$ \\
\hline
\end{tabular}

471-477, April 1993.

4] F.W. Singor and W M Snelgrove, "Switched-Capacitor Bandpass Delta-Sigma A/D Modulation at $10.7 \mathrm{MHz}$ " IEEE I Solid-State Circuits, Vol.30,pp. 184-192, March 1995.

[5] L. Longo and H.B. Horng, " $15 \mathrm{~b} 30 \mathrm{KHz}$ Bandpass Sigma-Delta Modulator," Proc. 1993 IEEE Int. Solid-State Circuit Conf., pp. 226-227, February. 1993.

[6] S.A. Jantzi, M. Snelgrove and P.F. Ferguson, "A Fourth-Order Bandpass Sigma-Delta Modulator," IEEE J. Solid-State Circuits, Vol. 28, pp. 282-291, March 1993

7] C.Toumazou, J.B.Hughes, and N.C. Battersby" "Switched Currents: An Analogue Technique for Digital Technology," London: Peter Peregrinus Ltd.,1993.

[8] N.Tan and S.Eriksson, "3.3V 11-bit Delta Sigma Modulator Using First-Generation Sl Circuits," Electron. Lett. Vol.30,pp. 20902091 , October 1994.

[9] M. Bracey, W. Redman-White and J.B.Hughes,"A SwitchedCurrent $\Sigma \Delta$ Converter for Direct Photodiode Interfacing," Proc 1994 IEEE Int. Symp. Circuits and Systems, pp. 287-290, May 1994.

[10] F.Medeiro, B. Pérez Verdú, A. Rodríguez Vázquez,and J.L. Huertas, "A Vertically-Integrated Tool for Automated Design of $\Sigma \Delta$ Modulators," IEEE Journal of Solid-State Circuits, Vol. 30, pp. 762-772, July 1995.

[11] M. Goldenberg, R. Croman and T. S. Fiez, "Accurate S| Filters Using RGC Integrators," IEEE J. Solid-State Circuits, Vol. 29, pp. 1388-1395, November 1994.

[12] S.J. Daubert and D. Vallancourt, "Operation and analysis of current copier circuits," IEEE Proc., Vol. 137, pp. 109-115, April 1990.

[13] M. Bracey and W. Redman-White, "Design Considerations for Current Domain Regenerative Comparators," Advanced A-D and D-A Conversion Techniques and their Applications, pp.65-70, July 1994.

[14] J.A. Svodoba, L. McGory and S. Webb, "Applications of a commercially available current conveyor,"Int. J. Electronics, Vol. 70, pp.159-164, January 1991.

[15] J. L. LaMay and H. J. Bogard, "How to obtain maximun practical performance from state-of-the-art Delta-Sigma analog-to-Digital converters," IEEE Trans. Instrument. Measure., vol 41., pp. 861867, Dec. 1992.

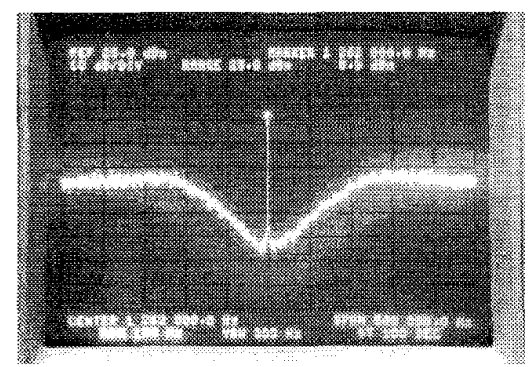

Fig. 15(a): Output spectrum of the integrated modulator $S N R\{d B$

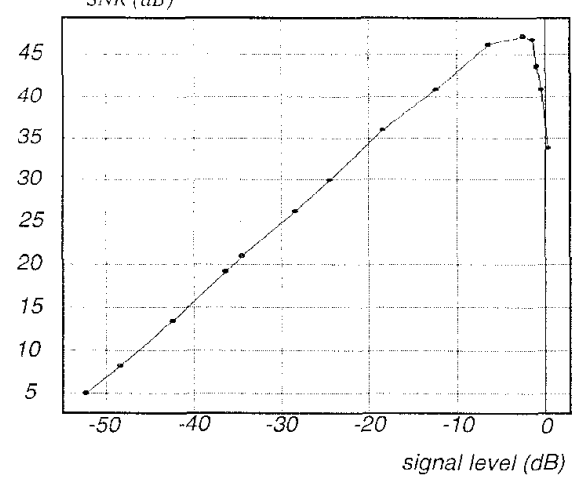

Fig. 15(b): Measured SNR vs. relative amplitude 